\title{
Spectral momentum density of graphite from $(e, 2 e)$ spectroscopy: Comparison with first-principles calculation
}

\author{
Chao Gao and A. L. Ritter \\ Department of Physics, Virginia Polytechnic Institute and State University, Blacksburg, Virginia 24061-4097 \\ J. R. Dennison \\ Department of Physics, University of Missouri at Columbia, Columbia, Missouri 65211 \\ N. A. W. Holzwarth \\ Department of Physics, Wake Forest University, P.O. Box 7507, Winston-Salem, North Carolina 27109
}

(Received 21 September 1987)

\begin{abstract}
We have measured the spectral momentum density $\rho(E, q)$ of graphite by $(e, 2 e)$ spectroscopy for momentum parallel and perpendicular to the crystal $c$ axis. In the independent-electron approximation, $\rho(E, \mathbf{q})=\Sigma_{\mathbf{G}}\left|U_{\mathbf{k}}(\mathbf{G})\right|^{2} \delta(\mathbf{q}-\mathbf{k}-\mathbf{G}) \delta(E-E(\mathbf{k}))$ where the one-electron wave function is $\Psi_{\mathbf{k}}(\mathbf{r})=e^{i \mathbf{k} \cdot \mathbf{r}} \Sigma_{\mathbf{G}} U_{\mathbf{k}}(\mathbf{G}) e^{i \mathbf{G} \cdot \mathbf{r}}$ and $\mathbf{G}$ is a reciprocal-lattice vector. The measurements covered a range of momentum parallel to the $c$ axis equal to $0 \leq|\mathrm{q}| \leq 1.84 \AA^{-1}$ and a range of momentum perpendicular to the $c$ axis equal to $0 \leq|\mathbf{q}| \leq 2.35 \AA^{-1}$. The energy range spanned the valence band of graphite from $4.4 \mathrm{eV}$ above the Fermi energy to $27.6 \mathrm{eV}$ below the Fermi energy. The momentum resolution was 0.47 and $0.73 \AA^{-1}$ (full width at half maximum) for momentum parallel and perpendicular to the $c$ axis, respectively. The energy resolution was $8.6 \mathrm{eV}$. The maximum coincidence rate was $\sim 0.02$ counts/sec. The band structure $E(\mathbf{k})$ and spectral density $\left|U_{\mathbf{k}}(\mathbf{G})\right|^{2}$ have been calculated from first principles using a self-consistent density-functional theory in the local-density approximation with a mixed-basis pseudopotential technique. The agreement within experimental uncertainties between measurement and theory is excellent.
\end{abstract}

\section{INTRODUCTION}

The spectral momentum density $\rho(E, q)$ of graphite has been measured by $(e, 2 e)$ spectroscopy for one direction of momentum in the basal plane $\left(0 \leq|\mathrm{q}| \leq 2.35 \AA^{-1}\right)$ and for momentum parallel to the $c$ axis $(0 \leq|\mathrm{q}| \leq 1.84$ $\AA^{-1}$ ). This is the first study of a crystal by this experimental technique. In the single-particle approximation the spectral density is equal to $\left|\Phi_{\mathbf{k}}(\mathbf{q}=\mathbf{k}+\mathbf{G})\right|^{2}$ when $E=E(\mathbf{k})$ ( $\mathbf{G}$ is a reciprocal-lattice vector). The spectral density of graphite has been calculated from first principles using self-consistent, density-functional theory in the local-density approximation with a mixed-basis pseudopotential technique. Excellent agreement is found within the experimental uncertainties between theory and the measurements reported here. The same theoretical approach has been used to calculate the band structure and charge density of graphite. ${ }^{1}$ The band structure has been measured by several groups using angle-resolved photoelectron spectroscopy (ARPES) for electron momentum in the basal plane of the crystal. ${ }^{2-11}$ The difference between theory and these measurements is less than or of order $1 \mathrm{eV}$. There is also good agreement between the calculated and measured charge densities. ${ }^{1}$ The densityfunctional technique has been used to calculate the ground-state properties of many solids and the agreement with measurements is often within a few percent. Thus, from our perspective, a mature theory of graphite exists and has been well confirmed by several independent experiments. We view the calculated spectral density as a benchmark for evaluating this new experimental technique, $(e, 2 e)$ spectroscopy. The agreement between our measurements and theory is strong evidence that the analysis $^{12,13}$ relating the $(e, 2 e)$ coincidence rate to the spectral momentum density is correct.

The spectral momentum density provides very detailed information about the electronic structure of solids and can be measured only by $(e, 2 e)$ spectroscopy. The band structure of crystalline solids can be measured by ARPES, but at this time information regarding the oneelectron wave functions cannot be obtained from the intensity of the photoemission peaks. The integral of $\rho(E, q)$ over momentum is the density of states, which, of course, can be measured by angle-integrated photoemission. The integral of $\rho(E, q)$ over energy, the momentum density, can be obtained from Compton scattering and positron annihilation. The price one pays to measure the full spectral momentum density by $(e, 2 e)$ spectroscopy is low count rate. The technique is a coincidence measurement, explained in more detail in the next section, and our maximum coincidence rate was approximately 1 event per minute. The experiment required four months of data taking. In order to obtain this rate, the energy resolution was $\Delta E=8.6 \mathrm{eV}$ [full width at half maximum (FWHM)] and the momentum resolution was estimated to be 0.47 and $0.73 \AA^{-1}$ (FWHM) for momentum parallel and perpendicular to the crystal $c$ axis, respectively. The coincidence rate is proportional to $\Delta E \Delta q^{4}$. Possible ways to increase the data rate are being explored, but it is clear that in general most information regarding the elec- 
tronic structure of crystalline solids can be obtained more expeditiously and with higher resolution by other techniques. Where $(e, 2 e)$ spectroscopy provides fundamental insights is in the investigation of disordered solids. For example, in an initial experiment on amorphous carbon, ${ }^{14}$ two well-defined bands were observed which did not broaden (within the experimental resolution) even well out beyond the momentum of the crystalline Brillouin zone boundary. This counterintuitive result is being investigated theoretically. ${ }^{15,16}$

The rest of this paper is organized as follows. The theory of $(e, 2 e)$ scattering from solids is discussed in the first section. The details of calculating the spectral momentum density of graphite are also described in that section. The experimental results are given in the second section and are analyzed in the third section. Finally, the results are summarized.

\section{THEORY}

The kinematics for $(e, 2 e)$ scattering is shown in Fig. 1(a). The energy of the incident electron is $25 \mathrm{keV}$. The angles of the scattered and recoiling electrons are $\theta_{1}=\theta_{2} \approx 45^{\circ}$ so that the energies of the outgoing electrons are $\sim 12.5 \mathrm{keV}$. The precollision binding energy and momentum of the ejected electron are determined by conservation of energy and momentum. Electrons in the target with momentum perpendicular to the incident beam direction can be observed by varying the angle $\phi$. Electrons with momentum parallel to the incident beam can be detected by varying $\theta_{1}$ and $\theta_{2}$ symmetrically about $45^{\circ}$. The cross section for $(e, 2 e)$ scattering from a target electron with binding energy $\varepsilon$ and real momentum $q$ is ${ }^{13}$

$$
\begin{aligned}
\frac{d^{4} \sigma}{d E_{1} d E_{2} d \Omega_{1} d \Omega_{2}}= & \left.\frac{m\left|\mathbf{P}_{1}\right|\left|\mathbf{P}_{2}\right|}{\hbar^{3}\left|\mathbf{P}_{0}\right|} \mid \frac{d \sigma}{d \Omega}\right]_{\text {Mott }} \\
& \times\left|F\left(\mathbf{q}=\mathbf{P}_{1}+\mathbf{P}_{2}-\mathbf{P}_{0}\right)\right|^{2} \\
& \times \delta\left(E_{1}+E_{2}-E_{0}-\varepsilon\right),
\end{aligned}
$$

where $(d \sigma / d \Omega)_{\text {Mott }}$ is the Mott electron-electron cross section which is a function of the scattering angle between $\mathbf{P}_{0}$ and $\mathbf{P}_{1}$. The function $|F(\mathbf{q})|^{2}$ is the momentum density of the one-electron target orbital $\Psi_{\alpha}(\mathbf{r})$

$$
F(\mathbf{q})=(1 / 2 \pi)^{3 / 2} \int e^{-\mathbf{i q} \cdot \mathbf{r}} \Psi_{\alpha}(\mathbf{r}) d \mathbf{r}
$$

where in a crystal, the one-electron orbital can be written as a Bloch wave function ( $\mathbf{G}$ is a reciprocal-lattice vector, $\mathbf{k}$ is a lattice wave vector, and $n$ is a band index)

$$
\Psi_{\mathbf{k}, n}(\mathbf{r})=e^{i \mathbf{k} \cdot \mathbf{r}} u_{\mathbf{k}, n}(\mathbf{r})=\sum_{\mathbf{G}} u_{\mathbf{k}, n}(\mathbf{G}) e^{i(\mathbf{k}+\mathbf{G}) \cdot \mathbf{r}}
$$

so that

$$
|F(\mathbf{q})|^{2}=\sum_{\mathbf{G}}\left|u_{\mathbf{k}, n}(\mathbf{G})\right|^{2} \delta(\mathbf{q}-\mathbf{k}-\mathbf{G}) .
$$

Thus, the spectral momentum density is a map of the band structure in the repeated zone representation with the intensity equal to the square of the Fourier expansion coefficient. In deriving the cross section, two approximations were made that require experimental verification. The first approximation is that the incident, scattered and recoiling electron can be represented by plane waves. There have been several studies of atomic and molecular systems ${ }^{17-19}$ which indicate that the plane-wave approximation is good when the incident electron energy is greater than $\sim 2 \mathrm{keV}$. The energy of the incident electron is an order of magnitude larger than this lower limit in our case so that the plane-wave approximation should be quite accurate. The second assumption, the impulse approximation, is that at large scattering angles and for incident energies much larger than the binding energy of the recoiling electron, the spectator electrons are frozen while the collision takes place. In other words, the single-particle states of all electrons except the incident and recoiling electrons do not change during the collision. This typically is not a good approximation in strongly correlated systems such as atoms, molecules, and narrow $d$ and $f$ bands of solids. In these cases "satellite" peaks to the single-particle spectrum are observed which arise from the excitation of spectator electrons during the collision process. These shake-up processes rarely occur in weakly correlated $S$ and $P$ valence bands of solids. Consistent with this general observation, we do not observe any structure in our $(e, 2 e)$ data which could be associated with multielectron excitations. The good agreement between our measurements and the spectral momentum density predicted on the basis of the singleparticle approximation is strong evidence that the approximations in the derivation of the $(e, 2 e)$ cross section are valid.

Finally, in our scattering geometry the proportionality constant between the spectral momentum density and the $(e, 2 e)$ coincidence rate is nearly independent of the target electron momentum $\mathbf{q}$, which is being observed. The proportionality constant does depend on $q$ because of the angular dependence of the Mott cross section, but the dependence is very weak. The Mott cross section changes by less than $6 \%$ as we sweep from $q=-3 \AA^{-1}$ to $3 \AA^{-1}$. Thus, to an excellent approximation the measured coincidence rate is a direct map of the spectral momentum density. It is difficult to make an absolute determination of the proportionality constant between coincidence rate and spectral momentum density, but it is the relative variation of $|F|^{2}$ with energy and momentum which is of primary interest and this quantity can be measured accurately.

The band structure of graphite has been calculated by several groups. ${ }^{1,20-27}$ Holzwarth, et al. used a firstprinciples, self-consistent technique which has been very successful in describing many ground-state properties of crystalline solids. The technique is based on densityfunctional theory in the local-density approximation using the mixed-basis pseudopotential approach. In addition to the band dispersion relations, the expansion coefficients for the band wave functions come out directly from these calculations. In Figs. 1(b) and 1(c) are shown the Brillouin zone of graphite and the band structure for particular symmetry directions. The six $\sigma$ valence bands, based on $s p^{2}$ orbitals in the basal plane, are shown as 
solid lines, while the two $\pi$ valence bands from the $p_{z}$ atomic orbitals are represented by dashed lines. Due to the weak interlayer interaction, the electronic structure is nearly two-dimensional, collapsing the number of valence bands to three $\sigma$ bands and one $\pi$ band. The spectral densities of the $\sigma$ and $\pi$ bands are shown in Fig. 2(a) for momentum in the basal plane $(\Gamma \rightarrow M)$. The wave functions for the lowest $\sigma$ bands are $S$-like; that is, the momentum density is maximum at $q=0$ and decreases monotonically as $q$ increases. The wave functions for the upper two $\sigma$ bands are $P$-like; the momentum density of the $\sigma_{2}$ band is zero at $q=0$, rises abruptly near the Brillouin zone boundary to a peak in the second zone, and then falls off exponentially as $q \rightarrow \infty$. The momentum density of the $\sigma_{3}$ band peaks in the third zone. The spectral densities in the $\Gamma \rightarrow K$ direction are shown in Fig. 2(b). They are qualitatively similar to the momentum densities in the $\Gamma \rightarrow M$ direction. The momentum density of the $\pi$ band is zero for $\mathrm{q}$ in the $\Gamma-K-M$ plane because the $\pi$ orbital has a node there. In Fig. 2(c) are the spectral momentum densities for $q$ along the $c$ axis in the $\Gamma-A$ direction. The bands are quite flat in this direction, dispersing less than or of order $1 \mathrm{eV}$. The momentum density of the lowest $\sigma$ band again falls off monotonically from $q=0$. The momentum densities of the upper $\sigma$ bands are zero for $\mathbf{q}$ along the $c$ axis for symmetry reasons. The momentum density of the $\pi$-band displays $p$-wave character; it is zero at the origin and peaks in the third zone at $q \approx 1.5 \AA^{-1}$. From these calculations one can see that graphite is an interesting blend of nearly free-electron behavior for $q$ in the crystal plane and atomic character for momentum perpendicular to the crystal plane.

\section{EXPERIMENTAL RESULTS}

The sample was natural graphite from mines at Ticonderoga, New York. Initial thinning of this layered material was by the "standard Scotch-tape method." We were not able to obtain usable samples less than $\sim 500 \AA$ thick by this procedure. In order to reduce the thickness to $\sim 100 \AA$ we took samples which were $\sim 1000 \AA$ thick and thinned them further by reactive ion etching using a mixture of oxygen and argon. There is evidence that plasma etching does far less damage to the surface layers of a crystal than ion milling. ${ }^{28}$ We obtained an area of film, slightly larger than the area of our electron beam spot, which we estimated was $\sim 150 \AA$ thick, based on the attenuation of a laser beam passing through the sample. From an analysis of the multiple scattering contribution to our elastic scattering data (described in the Ap-
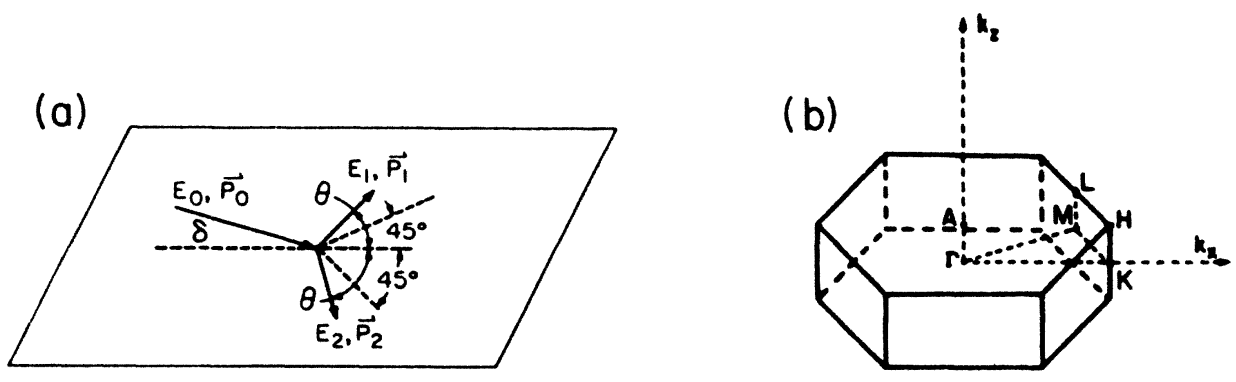

(c)

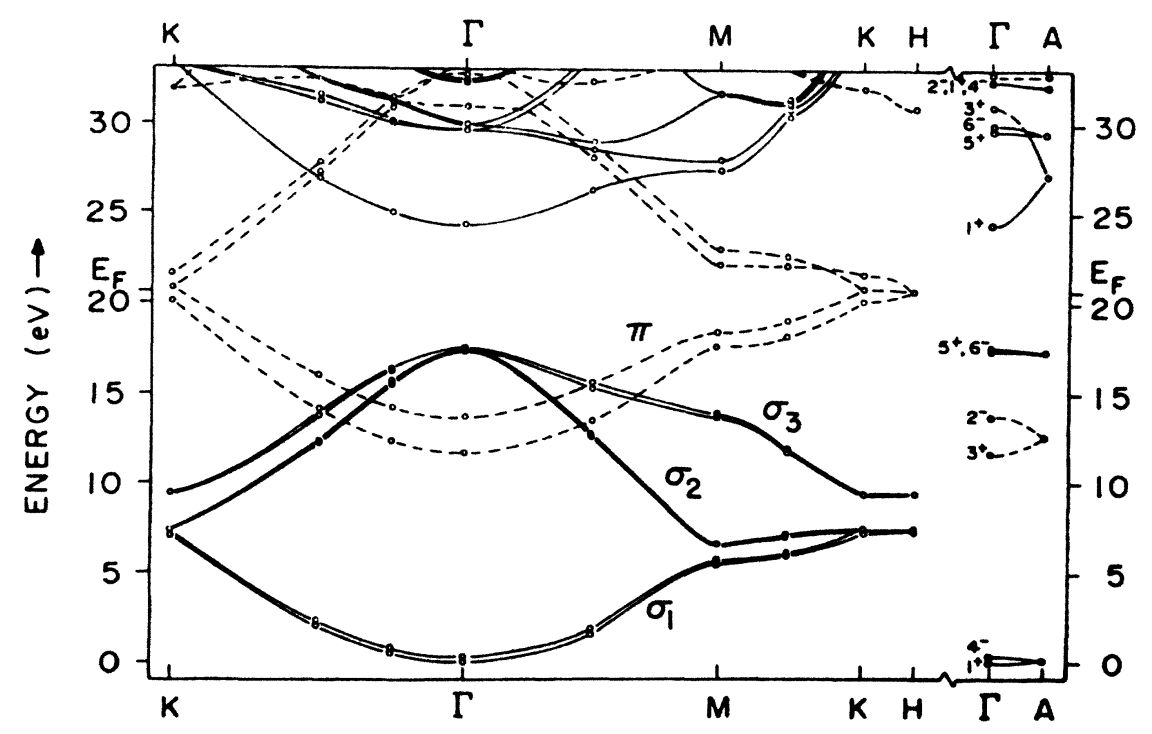

FIG. 1. (a) Kinematics for (e,2e) scattering. (b) Brillouin zone of graphite. (c) Band structure of graphite. 
pendix), we found the thickness was $\sim 110 \AA$, in rough agreement with the estimate by laser beam attenuation. The technique for preparing the film assured that the $c$ axis was perpendicular to the film surface. We attempted to measure the orientation of the crystal axes in the plane of the film, but were not successful. This is not too significant because with our present resolution the predicted variation in the spectral density for different momentum directions in the basal plane cannot be resolved.

In order to evaluate now much damage was done to the crystal by our thinning procedure, we measured the Raman spectra of the sample before and after etching it. We also checked the spectra after taking data for several weeks to see if the sample had been adversely affected by the electron beam. In Fig. 3(a) is the spectra of pristine graphite which exhibits a single Raman line at 1575 $\mathrm{cm}^{-1}$. After thinning sample with tape [Fig. 3(b)] and etching the sample [Fig. 3(c)], the $1575-\mathrm{cm}^{-1}$ line is still present and has not broadened. A new line at $1355 \mathrm{~cm}^{-1}$ is present which has been observed by others in microcrystalline graphite and arises from the breakdown of the

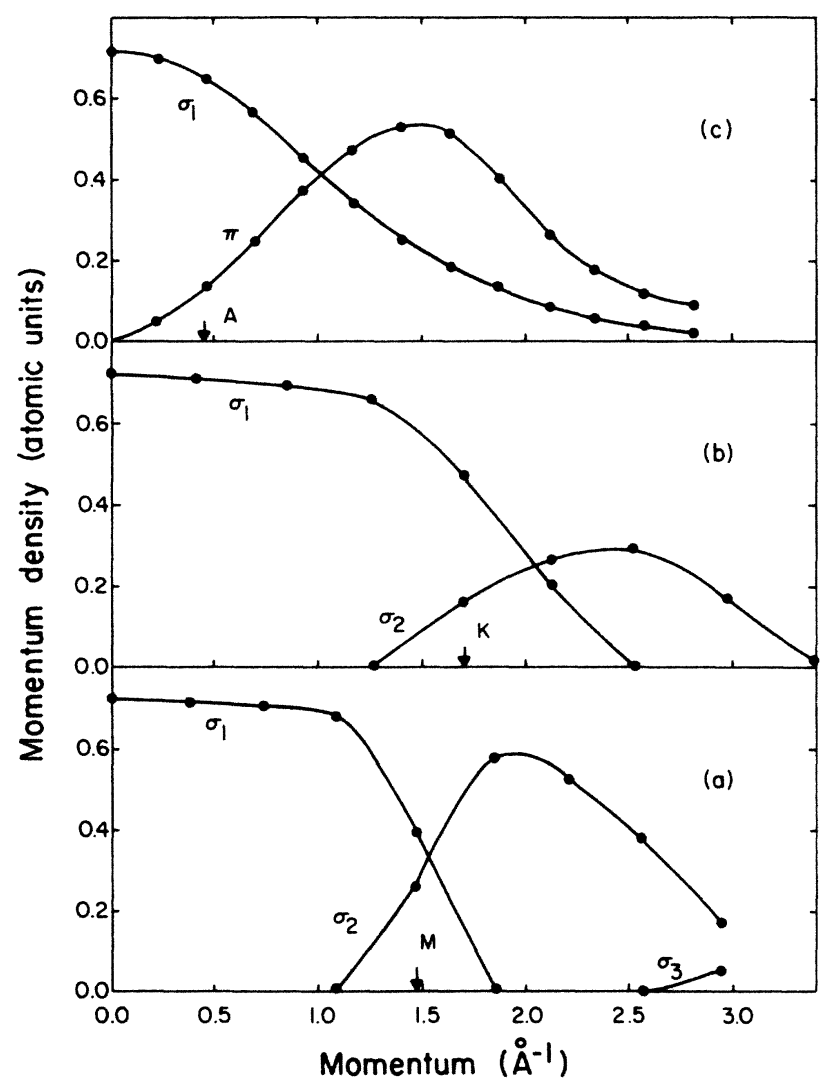

FIG. 2. Spectral momentum densities for different graphite bands as a function of momentum. (a) Momentum densities for $\sigma_{1}, \sigma_{2}$, and $\sigma_{3}$ bands for momentum in the $\Gamma-M$ direction. Momentum density of the $\pi$ band is zero by symmetry. (b) Momentum densities of the $\sigma_{1}$ and $\sigma_{2}$ bands for momentum in the $\Gamma-K$ direction. Momentum density of the $\pi$ band is zero by symmetry. (c) Momentum densities of the $\sigma_{1}$ and $\pi$ bands for momentum parallel to the $c$ axis $(\Gamma-A)$. Momentum densities of the $\sigma_{2}$ and $\sigma_{3}$ bands are zero by symmetry. $k$ selection rule as the crystallite size gets smaller. ${ }^{29-32}$ It has been observed that the relative intensity of the 1355 line with respect to the $1575-\mathrm{cm}^{-1}$ line varies inversely with the planar dimension of the crystallite. The small peak at $\sim 1622 \mathrm{~cm}^{-1}$ has been associated with weak disorder in the crystalline. In Fig. 3(d) is the Raman spectra of a sample after taking $(e, 2 e)$ data for five weeks. There is no visible change in the spectra. Finally, in Fig. 3(e) is the Raman spectra of amorphous carbon. It is clear that our sample has retained crystalline order through the process of thinning it.

The $(e, 2 e)$ spectrometer has been described in detail in Ref. 33. The incident electron energy was $25 \mathrm{keV}$ in these measurements. The energy resolution was $8.6 \mathrm{eV}$ FWHM and the momentum resolution was estimated to be 0.47 and $0.73 \AA^{-1}$ for momentum parallel and perpendicular to the $c$ axis, respectively. The coincidence

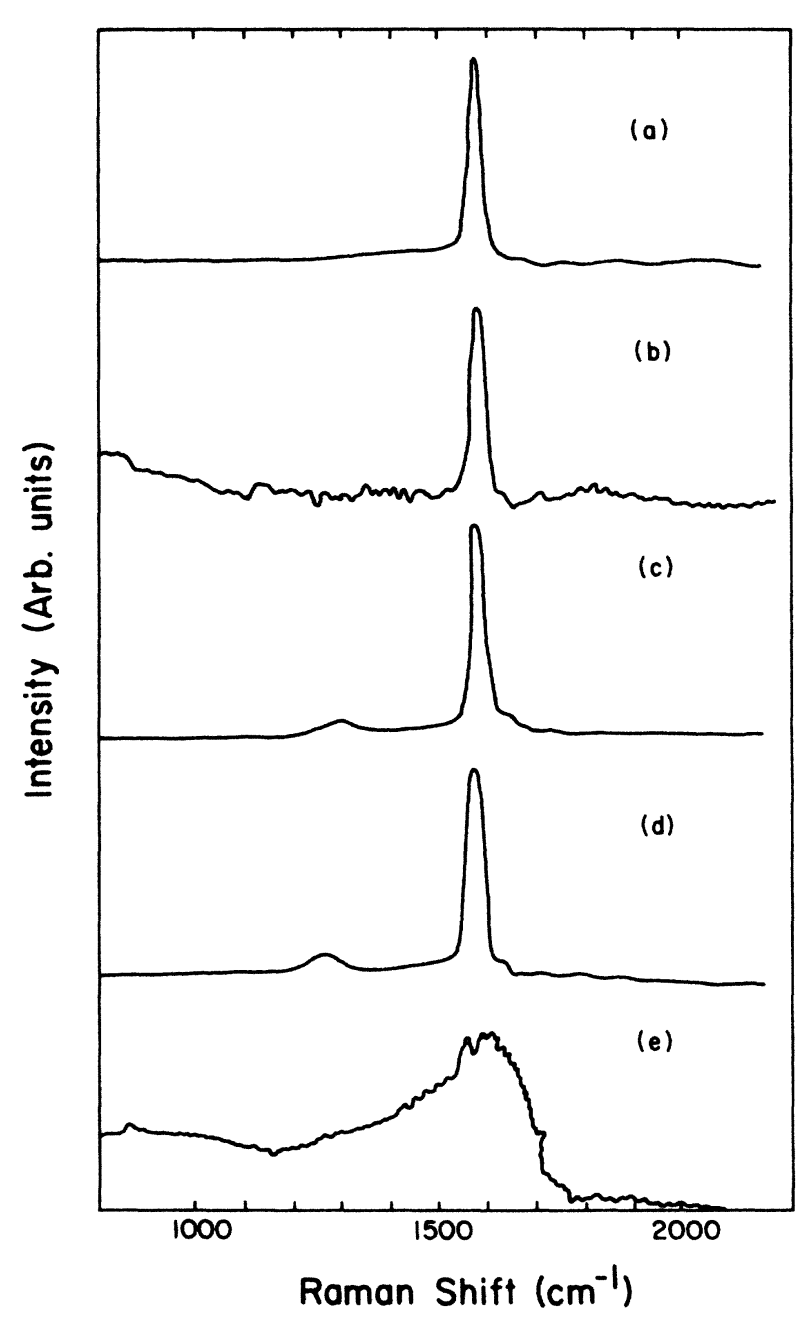

FIG. 3. Raman spectra of our samples and of amorphous carbon. (a) Raman spectra of graphite crystal. (b) Raman spectra of graphite film after thinning with scotch tape. (c) Raman spectra of graphite film after thinning with scotch tape and with reactive ion etching. (d) Raman spectra of graphite film after electron bombardment for five weeks. (e) Raman spectra of amorphous carbon. 
rate as a function of binding energy (with respect to the Fermi energy) for total momentum approximately equal to zero is shown in Fig. 4. The open circles are the raw data. Statistical error bars for one binding energy are shown.

The coincidence rate goes to zero above the Fermi energy, but does not go to zero below the bottom of the valence band ( $E_{B} \approx 20 \mathrm{eV}$ ) because of multiple scattering. A general procedure for deconvoluting the contributions of multiple scattering from the raw data has been given by Jones and Ritter. ${ }^{34}$ Specific application of this procedure to graphite, which is complicated by the crystalline anisotropy, is discussed in the Appendix. The solid circles in Fig. 4 are the deconvoluted $(e, 2 e)$ scattering rate. A weak peak at $\sim 8 \mathrm{eV}$ and a more intense peak at $\sim 20 \mathrm{eV}$ are clearly evident. The complete set of deconvoluted data is shown in Figs. 5(a) and 5(b). In Fig. 5(a) data are shown for the momentum vector in the basal plane and with the magnitude of the momentum ranging from -0.35 to $2.35 \AA^{-1}$ in steps of $0.3 \AA^{-1}$. The component of momentum parallel to the $c$ axis was nominally zero, but due to a misalignment of the spectrometer, the parallel offset was $\sim-0.61 \AA^{-1}$. In Fig. 5(b) data are shown for the momentum vector parallel to the $c$ axis from $-0.61 \AA^{-1}$ to $1.84 \AA^{-1}$ in steps of $0.27 \AA^{-1}$. Again, the perpendicular component of momentum was not zero, but was offset by $-0.35 \AA^{-1}$ because of misalignment. The solid lines in Figs. 5(a) and 5(b) are generated by fitting one or two Gaussian peaks to the data with the center and amplitude of the Gaussians as free parameters. The width of the Gaussian peaks was determined from the data near zero momentum. The momentum dependence of the width is negligible. In Tables I and II are the resulting parameters for momentum parallel and perpendicular to the $c$ axis, respectively. These results will be compared to theory in the next section.

The maximum coincidence rate we observed was $\approx 0.02$ counts/second for total momentum approximately zero and binding energy $\approx 20 \mathrm{eV}$. We find that multi-

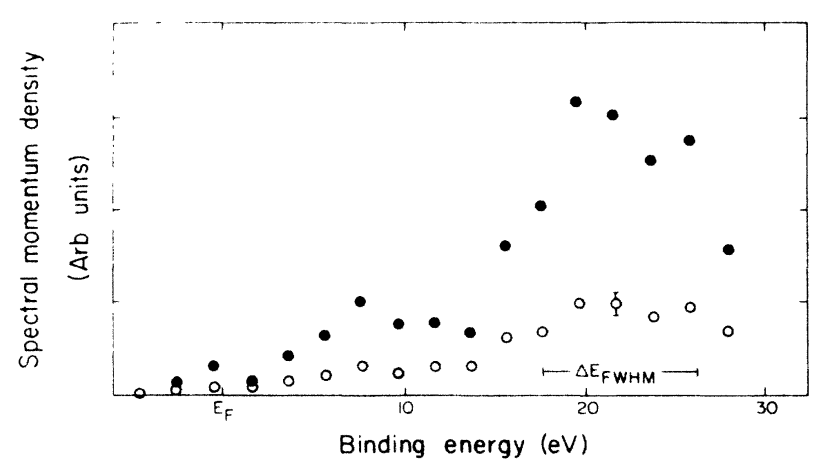

FIG. 4. Spectral momentum density of graphite as a function of energy (with respect to the Fermi energy) with the component of momentum parallel to the $c$ axis equal to $0.21 \AA^{-1}$ and with the component of momentum perpendicular to the $c$ axis equal to $-0.35 \AA^{-1}$. The open circles are the raw data. The solid circles are the data after correcting for multiple scattering. ple scattering is a major factor in determining the maximum rate. Coincidence events are removed from a particular energy-momentum bin by small-angle scattering (typically creation of a plasmon) either before or after the wide angle $(e, 2 e)$ collision. The optimum film thickness to maximize the coincidence rate is $\lambda / 2.7$ where $\lambda$ is the total mean free path of the incident electron. In the analysis of multiple scattering in our graphite data we found that the ratio of thickness to mean free path was 0.5 . The count rate would have been roughly $50 \%$ higher if the sample thickness had been optimum.

We took data over a four-month period. There are several sources of systematic errors which can arise over such a long collection time. We have considered (1) the incident electron current will vary, (2) the electron optics will vary affecting the incident current and the transmission of scattered electrons from the target to the detector, (3) high-frequency noise on the power or ground lines will produce bursts of false coincidence events, (4) the sample will change with time. The variation in beam current and tune conditions is mitigated by sweeping through a set of energy-momentum values in a period which is shorter than the drift time of the system. The energy-momentum set is swept repetitively in random order under computer control. After approximately $9 \mathrm{~h}$ a computer file is created which stores the coincidence data, and other information, for that period of time. Then all counters are

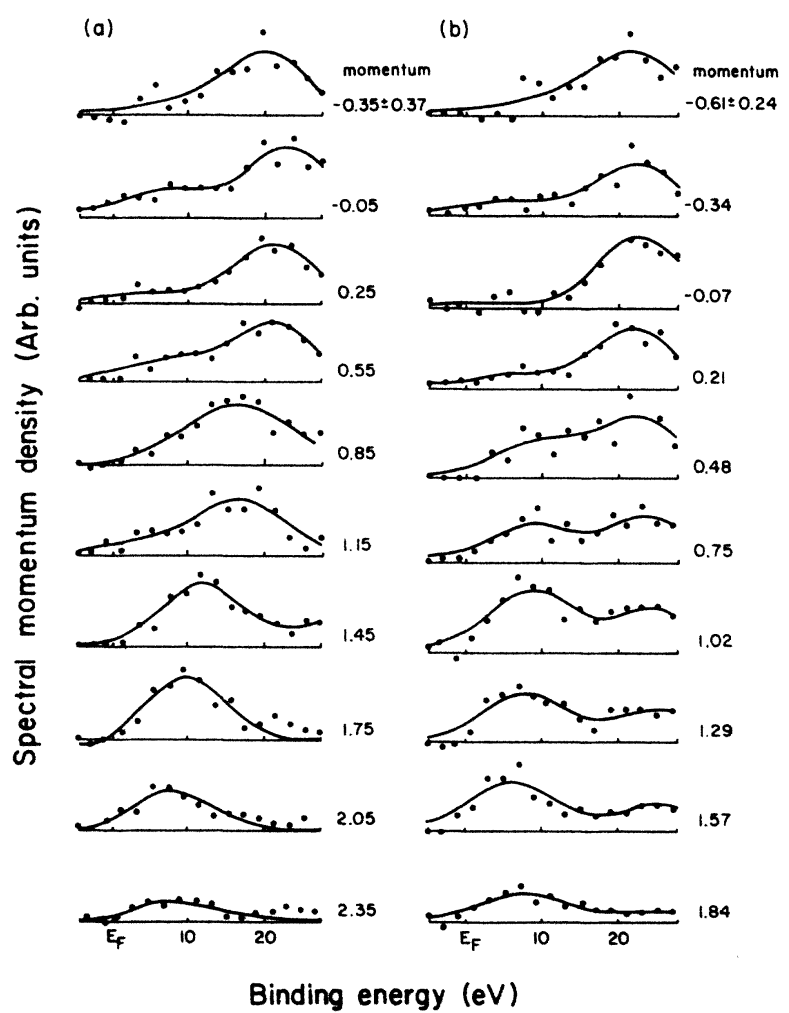

FIG. 5. The spectral momentum densities of graphite as a function of energy (with respect to the Fermi energy) for different momenta. The solid lines are least-squares fits of Gaussian peaks to the data. (a) Momentum perpendicular to the $c$ axis (parallel component is $-0.61 \AA^{-1}$ ). (b) Momentum parallel to the $c$-axis (perpendicular component is $-0.35 \AA^{-1}$ ). 
TABLE I. Momentum parallel to the $c$ axis (perpendicular component is $-0.35 \AA^{-1}$ ).

\begin{tabular}{lcccc}
\hline$q\left(\AA^{-1}\right)$ & $\begin{array}{c}\text { Energy } \\
\text { first peak }(\mathrm{eV})\end{array}$ & $\begin{array}{c}\text { Amplitude } \\
\text { first peak }\end{array}$ & $\begin{array}{c}\text { Energy } \\
\text { second peak (eV) }\end{array}$ & $\begin{array}{c}\text { Amplitude } \\
\text { second peak }\end{array}$ \\
\hline$-0.61 \pm 0.24$ & 10.65 & 0.12 & 22.3 & 0.56 \\
-0.34 & 6.06 & 0.11 & 20.9 & 0.47 \\
-0.07 & 2.94 & 0.04 & 22.5 & 0.67 \\
0.21 & 6.53 & 0.10 & 22.1 & 0.53 \\
0.48 & 9.79 & 0.31 & 22.9 & 0.53 \\
0.75 & 9.21 & 0.32 & 24.5 & 0.42 \\
1.02 & 9.32 & 0.55 & 25.3 & 0.40 \\
1.29 & 8.60 & 0.44 & 25.4 & 0.31 \\
1.57 & 6.85 & 0.42 & 26.2 & 0.23 \\
1.84 & 8.48 & 0.25 & 25.5 & 0.09 \\
\hline \hline
\end{tabular}

cleared and the process starts again. If there is evidence of false coincidence events in the file (the coincidence count is more than ten standard deviations from the average), due, for example, to high-frequency noise triggering the counter, then the file is rejected. Out of 174 files, we have rejected six. We monitor whether the sample is changing during the experiment by comparing files taken at the beginning of the run with files taken at the end. We saw no evidence of time dependence in the data. Finally, we took data in overlapping regions of binding energy and momentum rather than attempting to measure the total energy-momentum set in one block. This allowed us to better monitor the experiment and meant that complete data for some energy-momentum values would exist if the experiment aborted unexpectedly (the sample breaking, for example). The first set of data was taken on a course grid of momentum points, but spanned all the energy values. Then the other momentum columns were filled in. To normalize the data properly, we overlapped the later data sets with at least one momentum column from the first set. The ratio of the coincidence counts in the overlapping momentum columns was used as the normalizing factor.

\section{DISCUSSION}

Our data is compared with theory in Figs. 6 and 7 for momentum parallel and perpendicular to the $c$ axis, re- spectively. There is one free parameter for matching experiment and theory which is an overall scale factor. This factor has been determined from the average spectral density near $q=0\left(q \leq 0.5 \AA^{-1}\right)$ of the $\sigma_{1}$ band. The same scale factor is applied to all the data, for momentum both parallel and perpendicular to the $c$ axis. In the first case, Fig. 6, there is a constant component of momentum perpendicular to the $c$ axis, $q_{\perp}=-0.35 \AA^{-1}$, while the component of momentum parallel to the $c$ axis is variable. The $\pi$ band (dashed line) and $\sigma_{1}$ band (solid line), almost dispersionless along the $c$ axis, are drawn in the repeated zone representation. The dispersion is $\leq 1$ $\mathrm{eV}$ and has not been shown since it cannot be observed with our energy resolution. The first Brillouin-zone boundary for momentum parallel to the $c$ axis is $q_{\|}=0.47$ $\AA^{-1}$. We represent the spectral momentum density as an arrow on the energy-momentum plane. The theoretical densities are light arrows on top of the band dispersion; the experimental results from Table I are the heavy arrows. The agreement between theory and experiment is excellent for the $\pi$ band. The spectral density of the $\sigma_{1}$ band agrees very well with theory; the apparent dispersion of the band to larger binding energy is probably due to incomplete deconvolution of multiple scattering from the data. The spectral momentum densities of the two less tightly bound sigma bands, $\sigma_{2}$ and $\sigma_{3}$, are zero for symmetry reasons.

TABLE II. Momentum perpendicular to the $c$ axis (parallel component is $-0.61 \AA^{-1}$ ).

\begin{tabular}{lcccc}
\hline$q\left(\AA^{-1}\right)$ & $\begin{array}{c}\text { Energy } \\
\text { first peak (eV) }\end{array}$ & $\begin{array}{c}\text { Amplitude } \\
\text { first peak }\end{array}$ & $\begin{array}{c}\text { Energy } \\
\text { second peak (eV) }\end{array}$ & $\begin{array}{c}\text { Amplitude } \\
\text { second peak }\end{array}$ \\
\hline$-0.35 \pm 0.37$ & 7.10 & 0.08 & 20.1 & 0.57 \\
-0.05 & 7.49 & 0.19 & 23.0 & 0.60 \\
0.25 & 6.00 & 0.09 & 21.4 & 0.53 \\
0.55 & 7.71 & 0.16 & 21.3 & 0.50 \\
0.85 & 12.5 & 0.26 & 19.3 & 0.37 \\
1.15 & 4.64 & 0.12 & 17.2 & 0.48 \\
1.45 & & & 12.1 & 0.55 \\
1.75 & & & 9.20 & 0.62 \\
2.05 & & & 7.18 & 0.40 \\
2.35 & & & 8.53 & 0.19 \\
\hline \hline
\end{tabular}




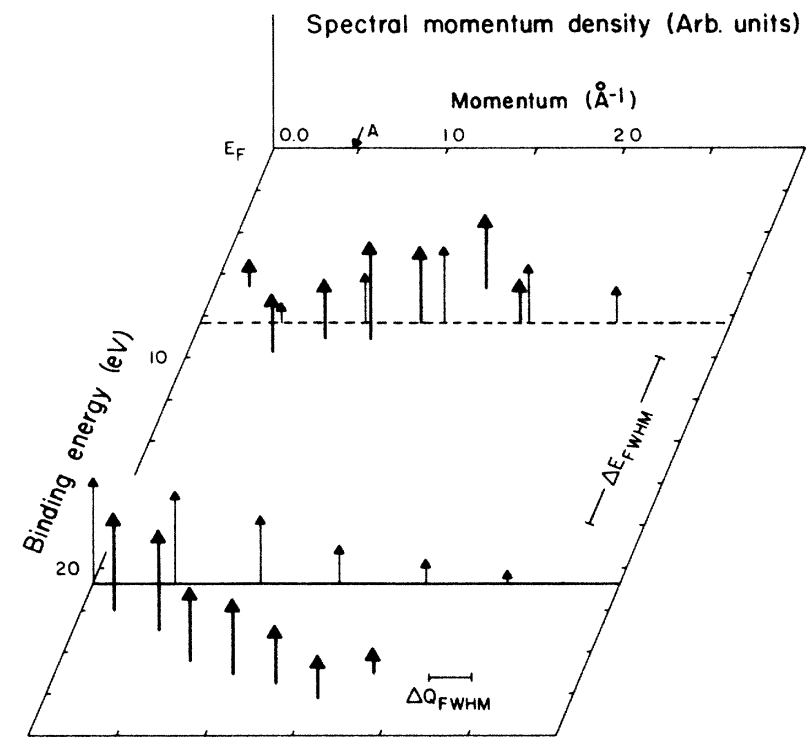

FIG. 6. The spectral momentum densities of graphite as a function of momenta. The momentum is parallel to the $c$ axis $(\Gamma-A)$ with a constant component perpendicular to the $c$ axis $q_{1}=-0.35 \AA^{-1}$. The dashed and solid lines are the $\pi$ and $\sigma_{1}$ bands, respectively. The light arrows are the theoretical spectral densities and the heavy arrows are the measured spectral densities from Table $\mathbf{I}$.

The spectral densities as a function of momentum perpendicular to the $c$ axis are shown in Fig. 7. In this case there is a constant component of momentum parallel to the $c$ axis $q_{\|}=-0.61 \AA^{-1}$. Again the theoretical density is represented by the light arrows placed on the energy dispersion curves, while the experimental results from Table II are represented by heavy arrows. There is very good agreement between theory and experiment for the $\sigma_{1}$ band. The spectral density of the $\sigma_{2}$ band is predicted to be negligible in the first Brillouin zone, then rise sharply at the zone boundary and peak in the second Brillouin zone. For $q \leq 1 \AA^{-1}$ we can not resolve the $\pi, \sigma_{2}$, and $\sigma_{3}$ bands because of our low resolution. We tentatively identify the weak structure at $q=0.25 \AA^{-1}(E=6 \mathrm{eV})$, $q=0.55 \AA^{-1}(E=7.7 \mathrm{eV})$, and $q=1.15 \AA^{-1}(E=4.6$ $\mathrm{eV}$ ) with the $\pi$ band. The existence of $\pi$ band spectral density is due to the small component of momentum parallel to the $c$ axis. The data at $q=0.85 \AA^{-1}$ can be fit almost equally well with two Gaussians centered at $E=12.5 \mathrm{eV}$ and $E=19.3 \mathrm{eV}$ (as plotted on Fig. 7) or with one Gaussian at $E=15.4 \mathrm{eV}$. The first choice preserves the continuity of the spectral density in the $\sigma_{1}$ band but raises the question of whether the peak at $E=12.5 \mathrm{eV}$ is associated with the $\sigma_{2}$ band. If it is, then the spectral density of the $\sigma_{2}$ band in the first Brillouin zone is much larger than predicted by theory. But this possible discrepancy is tentative until the measurement can be repeated with better resolution. In Fig. 7 we have compared our data to the calculated spectral density for momentum in the $\Gamma \rightarrow M$ direction. Within our resolution and statistics, the difference in spectral density for different directions in the basal plane cannot be resolved.

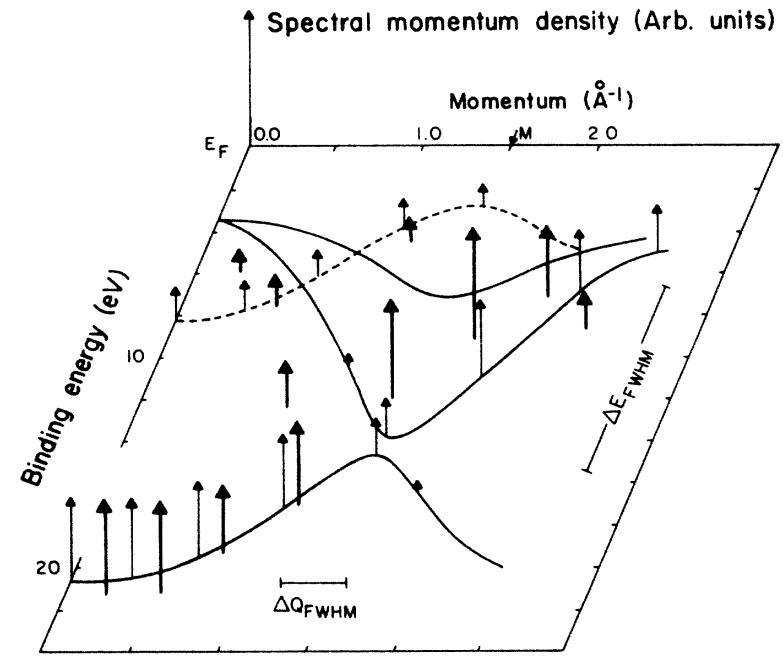

FIG. 7. The spectral momentum densities of graphite as a function of momenta and energy. The momentum is perpendicular to the $c$ axis (theoretical curves are for $\Gamma-M$ direction) with a constant component parallel to the $c$ axis $q_{\|}=-0.61$ $\AA^{-1}$. The dashed and solid lines are the $\pi$ and $\sigma_{1}$ bands, respectively. The light arrows are the theoretical spectral densities and the heavy arrows are the measured spectral densities from Table II.

\section{SUMMARY}

The spectral momentum density of graphite has been measured for momentum in the basal plane and for momentum parallel to the $c$ axis of the crystal. The results were compared to a first-principles calculation of the spectral density and excellent agreement was found. This is further confirmation of the power and accuracy of density-functional theory. Viewed from another perspective, graphite has been investigated by several experimental and theoretical techniques and the electronic structure of this material is understood well, certainly at the level of $\sim 1 \mathrm{eV}$ accuracy. The agreement between our measurements and the theoretical calculation of the spectral density provides evidence that the assumptions and approximations in the derivation of the $(e, 2 e)$ cross section are valid. The agreement further demonstrates that $(e, 2 e)$ spectroscopy gives sensible results when applied to a system which is well characterized. The technique can be extended with confidence now to other materials, such as amorphous solids, where the electronic structure is less well understood.

\section{ACKNOWLEDGMENTS}

We gratefully acknowledge the Raman measurements made by Mark Holtz. Samples were prepared in part at the National Nanofabrication Facility, which is supported by the National Science Foundation under Grant No. ECS-8619049, Cornell University, and industrial affiliates. This research was supported in part by Grant No. DMR-8420555 from the National Science Foundation. 


\section{APPENDIX}

In a typical $(e, 2 e)$ experiment on thin films, the target thickness is comparable to the mean free path for smallangle scattering. Jones and Ritter $^{34}$ have shown that the $(e, 2 e)$ coincidence rate $R$ for a film of thickness $\Delta$ can be written as the convolution of a "smearing function" $S$ and the differential inverse mean free path for $(e, 2 e)$ scattering, $d^{3} \Sigma_{(e, 2 e)} / d E d \Omega_{1} d \Omega_{2}$, which is the quantity of interest:

$$
R(\varepsilon, \mathrm{q})=\mathrm{I}_{0} \cdot \Delta \cdot \mathrm{S} \otimes \frac{d^{3} \Sigma_{(e, 2 e)}}{d E d \Omega_{1} d \Omega_{2}} \Delta E \Delta \Omega_{1} \Delta \Omega_{2}
$$

where " $\otimes$ " is the convolution operation, $I_{0}$ is the incident current (electrons/sec), and $\Delta E, \Delta \Omega_{1}$, and $\Delta \Omega_{2}$ are the experimental resolutions. The differential cross section is related to the differential inverse mean free path by

$$
\frac{d^{3} \sigma}{d E d \Omega_{1} d \Omega_{2}}=\frac{1}{\rho} \frac{d^{3} \Sigma}{d E d \Omega_{1} d \Omega_{2}},
$$

where $\rho$ is the density of target electrons. The smearing function incorporates all kinematically allowed combinations of small-angle, elastic, and inelastic scattering which can contribute to the multiple scattering background in the $(e, 2 e)$ coincidence data. The smearing function can be written

$S(\varepsilon, \mathrm{q} ; E, \Delta)$

$$
\begin{aligned}
\sum_{j_{e}, k_{e}, l_{e}, j_{i}, k_{i}, l_{i}=0}^{\infty} & C_{j_{e}, k_{e}, l_{e}, j_{i}, k_{i}, l_{i}}(E, \Delta) \\
& \times K_{j_{e}, k_{e}, l_{e}, j_{i}, k_{i}, l_{i}}(\varepsilon, \mathbf{q}),
\end{aligned}
$$

where $E$ is the incident energy, $C$ is defined in Ref. 34, and $K$ is a function of the probability for small angle scattering with total energy loss $\varepsilon$ and total momentum transfer q. The integer $j_{e}\left(k_{e}, l_{e}\right)$ is the number of elastic scattering events before the $(e, 2 e)$ collision [after the $(e, 2 e)$ collision for the scattered, recoiling electron] and $j_{i}$ $\left(k_{i}, l_{i}\right)$ is the number of inelastic scattering events before the $(e, 2 e)$ collision [after the $(e, 2 e)$ collision for the scattered, recoiling electron]. The function $K$ integrated over $\varepsilon$ and $q$ is normalized to unity. The sum of all the coefficients $C$ is also one. Thus, the term

$$
C_{j_{e}, k_{e}, l_{e}, j_{i}, k_{i}, l_{i}}(E, \Delta) K_{j_{e}, k_{e}, l_{e}, j_{i}, k_{i}, l_{i}}(\varepsilon, \mathbf{q})
$$

in the function $S$ is the probability that $j_{e}+k_{e}+l_{e}$ elastic scattering events and $j_{i}+k_{i}+l_{i}$ inelastic scattering events will occur in a film of thickness $\Delta$ when the energy of the incident beam is $E$, the total energy loss is $\varepsilon$, and the total momentum transfer is q. The function $K$ is

$$
K_{j_{e}, k_{e}, l_{e}, j_{i}, k_{i}, l_{i}}=P_{j_{e}, \mathrm{el}} \otimes P_{j_{i}, \text { in }} \otimes P_{k_{e}, \mathrm{el}}^{\prime} \otimes P_{k_{i}, \text { in }}^{\prime} \otimes P_{l_{e}, \mathrm{el}}^{\prime \prime} \otimes P_{l_{i}, \text { in }}^{\prime \prime}
$$

where

$$
P_{n, \mathrm{el}}=\lambda_{\mathrm{el}} \frac{d^{4} \Sigma_{\mathrm{el}}}{d^{3} q d \varepsilon} \otimes P_{n-1, \mathrm{el}},
$$

$P_{0, \mathrm{el}}$ is the resolution function of the incident beam,

$$
\begin{aligned}
& P_{n, \text { in }}=\lambda_{\text {in }} \frac{d^{4} \Sigma_{\text {in }}}{d^{3} q d E} \otimes P_{n-1, \text { in }}, \\
& P_{0, \text { in }}=\delta(\mathbf{q}) \delta(E),
\end{aligned}
$$

where

$$
d^{4} \Sigma_{\mathrm{el}} / d^{3} q d \varepsilon=\left(d^{3} \Sigma_{\mathrm{el}} / d^{3} q\right) \delta(E)
$$

and $d^{4} \Sigma_{\text {in }} / d^{3} q d \varepsilon$ are the differential inverse mean free paths for elastic and inelastic scattering, respectively. The factors $\lambda_{\mathrm{el}}$ and $\lambda_{\mathrm{in}}$ are the mean free paths for elastic and inelastic scattering. The functions $P^{\prime}$ and $P^{\prime \prime}$, referring to the scattered and recoiling electrons, are the same as the function $P$ except the energy of the electrons are roughly half the energy of the incident electrons and the coordinate system for the momentum is different for the incident, scattered, and recoiling electrons. Thus, the smearing function is determined by four functions: $d^{4} \Sigma_{\mathrm{el}} / d^{3} q d \varepsilon, d^{4} \Sigma_{\text {in }} / d^{3} q d \varepsilon, P_{0, \mathrm{el}}$ and $P_{0, \mathrm{el}}^{\prime}$ (in our spectrometer the resolution function for the scattered and recoiling electrons, $P_{0, \text { el }}^{\prime}$ and $P_{0, \text { el }}^{\prime \prime}$ are equal). There are several ways to deconvolute $d^{3} \Sigma_{(e, 2 e)} / d E d \Omega_{1} d \Omega_{2}$ from the measured coincidence rate. We have used the iterative technique suggested by Van Cittert: ${ }^{35}$

$$
\begin{aligned}
& \Sigma_{(e, 2 e)}^{(1)}=2 R / \Delta I_{0}-R \otimes S / \Delta I_{0}, \\
& \Sigma_{(e, 2 e)}^{(n)}=\Sigma_{(e, 2 e)}^{(n-1)}+R / \Delta I_{0}-\Sigma_{(e, 2 e)}^{(n-1)} \otimes S,
\end{aligned}
$$

where

$$
\Sigma_{(e, 2 e)}=\left(d^{3} \Sigma_{(e, 2 e)} / d E d \Omega_{1} d \Omega_{2}\right) \Delta E \Delta \Omega_{1} \Delta \Omega_{2} .
$$

We discuss next the differential inverse mean free path for elastic scattering and then the differential inverse mean free path for inelastic scattering. With these functions, we have calculated $S$ up to second-order multiple scattering. We estimate how the cutoff at second order affects the accuracy of $\Sigma_{(e, 2 e)}$.

Elastic scattering will occur only if the Bragg condition is satisfied. In our scattering geometry to observe target electron momentum $q$ in the plane of the film we vary the angle of the incident beam with respect to the normal of the film [see Fig. 1(a)]. For our graphite sample the $c$ axis is normal to the plane of the film. There will be no Bragg scattering when observing electrons with momentum inside the first Brillouin zone. The incident beam will be elastically scattered when the component of the momentum perpendicular to the $c$ axis first touches the Brillouin zone boundary $\left(q_{1} \approx 1.5 \AA^{-1}\right)$. The Bragg reflection changes the sign of the angle between the incident electron and the $c$ axis but does not affect the magnitude of $q_{\perp}$ which is observed. Since the band dispersion and spectral momentum density are invariant under the transformation $\mathbf{q} \rightarrow-\mathbf{q}$, the $(e, 2 e)$ data are not affected by Bragg scattering of the incident beam. The scattered and recoiling electrons leaving the $(e, 2 e)$ vertex also can be Bragg reflected before they exit from the sample. There will be no scattering of these two electrons until the component of momentum parallel to the $c$ axis is $q_{\|} \approx 2.9$ $\AA^{-1}$. This is nearly 6 times the momentum of the first 
Brillouin zone boundary in the direction of the $c$ axis and lies beyond our last data point at $q=1.84 \AA^{-1}$. Thus, for momenta perpendicular and parallel to the $c$ axis of graphite our $(e, 2 e)$ data was unaffected by elastic Bragg scattering.

The smearing function to second order contains contributions only from small-angle, inelastic scattering of the incident, scattered, and recoiling electrons. Since graphite is anisotropic, the differential inverse mean free path for small-angle scattering is a tensor which depends on the initial and final direction of the scattered electron. The tensor is diagonal in a coordinate system in which momentum is measured relative to the $c$ axis. The differential inverse mean free path written in terms of the dielectric function is ${ }^{36}$

$$
\begin{aligned}
& \frac{d^{4} \Sigma_{\mathrm{in}}(E, \mathbf{q})}{d^{3} q d E} \\
& \approx\left(1 / a_{0} E_{0}\right) \operatorname{Im}\left(\frac{-1}{q^{2} \cos ^{2} \theta \varepsilon_{\|}(E, \mathbf{q})+q^{2} \sin ^{2} \theta \varepsilon_{1}(E, \mathbf{q})}\right) \\
& \quad \times \delta\left(\mathbf{q} \cdot \mathbf{v}-\hbar \omega_{p}\right),
\end{aligned}
$$

where $a_{0}$ is the Bohr radius, $E_{0}$ is the energy loss, $E_{0}$ is the incident energy, $\theta$ is the angle between $q$ and the $c$ axis, $\varepsilon_{\|}$is the dielectric function for $q$ parallel to the $c$ axis, $\varepsilon_{1}$ is the dielectric function for $\mathbf{q}$ perpendicular to the $c$-axis, $\omega_{p}$ is the plasmon energy, and $v$ is the incident velocity. Measurements ${ }^{37}$ of $d^{4} \Sigma_{\text {in }} / d^{3} q d \varepsilon$ as a function of $|\mathbf{q}|$ show that it is proportional to $1 / q^{2}$ out to a critical momentum $q_{c} \approx 1.25 \AA^{-1}$, then it falls off more rapidly than $1 / q^{2}$. We have made the approximation that $\varepsilon_{\|}$ and $\varepsilon_{1}$ are independent of $q$ for $q \leq q_{c}$ and that $d^{4} \Sigma_{\text {in }} / d^{3} q d \varepsilon$ is zero for $q>q_{c}$. For the energy dependence of the parallel and perpendicular dielectric function we fit the experimental data of Ref. 38 to the Drude-Sellmeier formula. ${ }^{39}$ The perpendicular dielectric function was fit with a plasma energy of $21 \mathrm{eV}$ and two harmonic oscillators at 15 and $4 \mathrm{eV}$. The damping constants [ $\hbar /($ relaxation time)] were $3.0,7.0$, and $2.0 \mathrm{eV}$ for the plasmon and two harmonic oscillators, respectively. The oscillator strengths were $0.15,0.80$, and 0.15 , respectively. The parallel dielectric function was fit with a plasmon energy of $15.0 \mathrm{eV}$ and three harmonic oscillators at 15.0, 10.0, and $4.0 \mathrm{eV}$. The respective damping constants were $1.5,12.0,0.75$, and $2.0 \mathrm{eV}$. The respective oscillator strengths were $0.35,0.50,0.20$, and 0.05 . These parameters gave an excellent fit for the positions of the small-angle, energy-loss peaks. The experimental and fitted peak heights differ by less than $3 \%$ for momentum parallel to the $c$ axis and $12 \%$ for momentum perpendicular to the $c$ axis.

The last parameters which enter the smearing function are the target thickness and the mean free path for inelastic scattering. These parameters always appear as a ratio in the smearing function. This ratio can be determined from the multiple scattering peaks associated with elastic scattering of electrons at $45^{\circ}$. In addition to the elastic peak there are peaks at lower energy corresponding to electrons which have created plasmons either before or after the $\sim 45^{\circ}$ elastic vertex. The ratios of the plasmon peaks to the elastic peak can be calculated in the same way as the smearing function. Assuming the elastic mean free path for graphite is infinite, the ratio of the first plasmon loss peak to the elastic peak is

$$
\begin{aligned}
& \frac{I_{\text {one-plasmon }}}{I_{\text {elastic peak }}} \\
& =\frac{\Delta}{\lambda_{\text {in }}} \int g(\beta) \int d \mathrm{q} \lambda_{\text {in }} \frac{d^{4} \Sigma_{\text {in }}}{d^{3} q d E} \otimes \frac{F\left(E=E_{p}, \mathrm{q}\right)}{\Delta q_{x} \Delta q_{y} \Delta q_{z}} \\
& \left.\quad+\sqrt{2} h(\beta) \int d \mathrm{q} \lambda_{\text {in }} \frac{d^{4} \Sigma_{\text {in }}^{\prime}}{d^{3} q d \varepsilon} \otimes \frac{F\left(E=E_{p}, \mathrm{q}\right)}{\Delta q_{x} \Delta q_{y} \Delta q_{z}}\right), \\
& g(\beta)=1-1 / \beta+1 /\left(e^{\beta}-1\right), \\
& h(\beta)=1+1 / \beta-e^{\beta /\left(e^{\beta}-1\right),} \\
& \beta=(\sqrt{2}-1) \Delta / \lambda_{\text {in }},
\end{aligned}
$$

where $d^{4} \Sigma_{\text {in }} / d^{3} q d \varepsilon$ and $d^{4} \Sigma_{\text {in }}^{\prime} / d^{3} q d \varepsilon$ are the differential loss functions before and after the $45^{\circ}$ elastic scattering event, respectively. The resolution function $F(E, \mathbf{q})=f(E) f(\mathbf{q})$ is defined such that

$$
\begin{aligned}
& f(E=0)=f(\mathbf{q}=0)=1, \\
& \int f(E) d E=\Delta E, \\
& \int f(\mathbf{q}) d \mathbf{q}=\Delta q_{x} \Delta q_{y} \Delta q_{x} .
\end{aligned}
$$

Fitting the one parameter, $\Delta / \lambda_{\text {in }}$, to our data we find $\Delta / \lambda_{\text {in }}=0.5$ for electrons of energy $25 \mathrm{keV}$. The inelastic mean free path is $\lambda_{\text {in }}=220 \AA$, from integrating $d^{4} \Sigma_{\text {in }} / d^{3} q d E$. This implies that $t=110 \AA$, which is consistent with our estimate of the thickness from the attenuation of light.

In the deconvolution of our data, only first- and second-order terms in the smearing function were calculated. The first-order term is the $(e, 2 e)$ vertex with no other scattering. The second-order term is an $(e, 2 e)$ vertex with one small-angle, inelastic vertex for either the incident, scattered, or recoiling electron. In the deconvolution procedure, the second-order term required numerical integration of a four-dimensional integral. A third-order term would require numerical evaluation of a sixdimensional integral. We have estimated the error in our deconvolution procedure from dropping higher-order terms in the smearing function by the following argument. Assume the differential inverse mean free path for $(e, 2 e)$ scattering is zero for binding energies less than the Fermi energy, and constant (say $\Sigma^{0}$ ) for binding energies greater than the Fermi energy. Then it is straightforward to show that the measured coincidence rate at binding energy, $E_{B}$, is $\Sigma^{0}$ times the integral of the smearing function over energy and momentum with the upper limit on the energy integral equal to $E_{B}$. The first-order term in the smearing function peaks near $E_{B}=0$ (the Fermi energy); the second-order term peaks near the plasmon energy $(\sim 25 \mathrm{eV})$; the third-order term peaks at twice the plasmon energy; and higher order terms peak at even larger binding energies. Our data extend from 
$E_{B}=-4.4 \mathrm{eV}$ to $E_{B}=27.6 \mathrm{eV}$, so that only the first- and second-order terms in the smearing function influence significantly the measured coincidence rate. We have estimated the error due to cutting off the series at second order by integrating the first-, second-, and third-order terms in the smearing function over the range of our data. The third-order contribution is $\sim 6 \%$ of the firstand second-order contribution.
${ }^{1}$ N. A. W. Holzwarth, Steven G. Louie, and Shrab Rabii, Phys. Rev. B 26, 5382 (1982).

${ }^{2}$ A. Bianconi, S. B. M. Hagström, and R. Z. Bachrach, Phys. Rev. B 16, 5543 (1977).

${ }^{3}$ W. Eberhardt, I. T. McGovern, E. W. Plummer, and J. E. Fisher, Phys. Rev. Lett. 44, 200 (1980).

${ }^{4}$ A. R. Law, M. T. Johnson, and H. P. Hughes, Phys. Rev. B 34, 4289 (1986).

${ }^{5}$ F. R. McFeely, S. P. Kowalczyk, L. Ley, R. G. Cavell, R. A. Pollak, and D. A. Shirley, Phys. Rev. B 9, 5268 (1974).

${ }^{6}$ D. Marchand, C. Fretigny, M. Lagues, F. Batallan, Ch. Simon, I. Rosenman, and R. Pinchaux, Phys. Rev. B 30, 4788 (1984).

${ }^{7}$ T. Takahshi, H. Tokailin, and T. Sagawa, Solid State Commun. 52, 765 (1984).

${ }^{8}$ A. R. Law, J. J. Barry, and H. P. Hughes, Phys. Rev. B 28, 5332 (1983).

${ }^{9}$ I. T. McGovern, W. Eberhardt, E. W. Plummer, and J. E. Fisher, Physica B + C 99B, 415 (1980).

${ }^{10}$ P. M. Williams, D. Latham, and J. Wood, J. Electron Spectrosc. Relat. Phenom. 7, 281 (1975).

${ }^{11}$ R. F. Willis, B. Feuerbacher, and B. Fitton, Phys. Rev. B 4, 2441 (1971).

${ }^{12}$ I. E. McCarthy and E. Weigold, Rep. Prog. Phys. (to be published).

${ }^{13}$ V. G. Levin, V. G. Neudachin, and Yu. F. Smirnov, Phys. Status Solidi B 49, 489 (1972).

${ }^{14}$ A. L. Ritter, J. R. Dennison, and R. Jones, Phys. Rev. Lett. 53, 2054 (1984).

15J. L. Beeby and T. M. Hayes, Phys. Rev. B 32, 6464 (1985).

${ }^{16}$ R. Haydock (unpublished).

${ }^{17}$ E. Weigold and I. E. McCarthy, Adv. At. Mol. Phys. 14, 127 (1978).

${ }^{18}$ K. T. Leung and C. E. Brion, J. Electron Spectrosc. Relat. Phenom. 35, 327 (1985).

${ }^{19} \mathrm{G}$. Stefani, R. Camilloni, and A. Giardini Guidoni, Phys. Lett.
64A, 364 (1978).

${ }^{20}$ H. J. F. Jansen and A. J. Freeman, Phys. Rev. B 35, 8207 (1987).

${ }^{21}$ R. C. Tatar and S. Rabii, Phys. Rev. B 25, 4126 (1982).

${ }^{22}$ A. Zunger, Phys. Rev. B 17, 626 (1978).

${ }^{23}$ A. Bianconi, S. B. M. Hagström, and R. Z. Bachkrach, Phys. Rev. B 16, 5543 (1977).

${ }^{24}$ H. Nagayoshi, K. Nakao, and Y. Uemora, J. Phys. Soc. Jpn. 41, 1480 (1976).

${ }^{25}$ R. F. Willis, B. Fitton, and G. S. Painter, Phys. Rev. B 9, 1926 (1974).

${ }^{26}$ I. L. Spain, in Chemistry and Physics in Carbon, edited by P. L. Walker and P. A. Thrower (Dekker, New York, 1973), Vol. 8, p. 1.

${ }^{27}$ G. S. Painter and D. F. Ellis, Phys. Rev. B 1, 4747 (1970).

${ }^{28}$ J. Dieleman and F. H. M. Sanders, Solid State Technol. 27, 191 (1984).

${ }^{29}$ R. O. Dillon, John A. Wollam, and V. Kathanant, Phys. Rev. B 29, 3482 (1982).

${ }^{30}$ P. Lespade, R. Al-Jishi, and M. S. Dresselhaus, Carbon 20, 427 (1982).

${ }^{31}$ R. J. Nemanich and S. A. Solin, Phys. Rev. B 20, 392 (1979).

${ }^{32}$ F. Tuinstra and J. L. Koenig, J. Chem. Phys. 53, 1126 (1970).

${ }^{33}$ A. L. Ritter, J. R. Dennison, and J. Dunn, Rev. Sci. Instrum. 55, 1280 (1984)

${ }^{34}$ R. Jones and A. L. Ritter, J. Electron Spectrosc. Relat. Phenom. 40, 285 (1986).

${ }^{35}$ P. H. Van Cittert, Z. Phys. 69, 298 (1931).

36J. Daniels, C. Von Feslenberg, H. Raether, and K. Zepperfeld, in Springer Tracts in Modern Physics, edited by G. Hohler (Springer, Berlin, 1970), Vol. 54, p. 77.

${ }^{37}$ K. Zeppenfeld, Z. Phys. 211, 391 (1968).

${ }^{38}$ H. Venghaus, Phys. Status Solidi B 71, 609 (1975).

${ }^{39}$ C. J. Powell, J. Opt. Soc. Am. 59, 738 (1969). 